\title{
Analysis of Linkage Mechanisms with Internal Driving Link
}

\author{
Mykhaylo Pashechko', Viacheslav Pasika², Nataliya Hembara³, \\ Viacheslav Kharzhevskyi ${ }^{4}$
}

1 Department of Fundamentals of Technology, Lublin University of Technology, 38A Nadbystrzycka str., 20-618 Lublin, Poland

2 Department of Technical Mechanics and Dynamics of Machines, Lviv Polytechnic National University, 12 Bandera str., 79013, Lviv, Ukraine

${ }^{3}$ Department of Engineering Mechanics, Ukrainian Academy of Printing, 19 Pidgolosko str., 79020, Lviv, Ukraine

${ }^{4}$ Department of Industrial and Agricultural Engineering, Khmelnytskyi National University, 11 Instytutska str., 29016, Khmelnytskyi, Ukraine

* Corresponding author's e-mail: mpashechko@hotmail.com

\begin{abstract}
The linkage mechanisms with internal driving link that are used in many different machines are considered in the paper. The mechanism contains hydraulic cylinder that is hinged to the rack, a rocker arm that is pivotally attached to the driving rod and to the rack. The law of motion of a rod in a hydraulic cylinder was synthesized. The positions, angular velocities and accelerations of the hydraulic cylinder and the rocker arm are analytically determined by the methods of engineering mechanics and the theory of mechanisms and machines. Thus, brief analytical dependencies are obtained. It is shown that the angular accelerations of the hydraulic cylinder and the rocker arm are qualitatively similar to the relative acceleration of the rod. The results of the work can be used in the motion research of hydraulically driven excavator buckets, other lifting and loading, road building machines, in the actuating mechanisms of industrial robots.
\end{abstract}

Keywords: non-Assur structural group, internal driving link, excavators, wheel cranes, hydraulic drive.

\section{INTRODUCTION}

Linkage mechanisms with an internal driving link are used as actuators for industrial robots, in lifting and loading, road building machines, in simulators, in training devices, etc. They are used as main mechanisms of excavators with hydraulic drive, which are produced by different companies, for example CAT, Volvo, Doosan, Hitachi, Atlas, JCB and others.

The kinematic analysis and synthesis of linkage mechanisms with the primary link acting as an input link, is not difficult and is described in many papers on Theory of Mechanisms and Machines [3, 5-7]. However, the case of the internal driving link is much more complicated, so there are few studies dedicated to the partial cases that are available on that topic. But without kinematic analysis of such mechanisms it is impossible to obtain the dynamic characteristics of hydraulically driven excavators and to make proper conclusions about the design of adaptive operation methods. Therefore, the kinematic analysis is the first and important stage in the overall design process of linkage mechanisms with internal driving link that are used in many machines.

The mechanisms with the internal driving link cannot be classified by well-known Assur classification, so they are called as non-Assur structural groups [1, 11]. Degrave V. in the work [1] determined only special positions of non-Assur structural groups, without the calculation of the velocities and accelerations of the links, Terenteva A. in the work [11], using excavator CHETRA 
EGP-320 as an example, considered the problem of positioning accuracy of an excavator bucket, which kinematic chain contains three drive modules (non-Assur groups). On the whole, the problem of analysis of the mechanisms with nonAssur structural groups has not been solved yet, although the mechanism that is considered in the article is the main one in the hydraulically driven bucket excavators. Therefore, it is an important task to develop methods of calculation of kinematic and dynamic characteristics of the mechanisms with non-Assur structural groups.

The goal of the paper is to research the motion of the links of an example non-Assur structural group, which can be used as a part of various lifting and loading machines, at the given movement of the internal driving link. Besides, the task is to determine the influence of the motion law of the driving link on the movement of the other links of the mechanism.

\section{ANALYSIS OF THE MECHANISM}

The drive module, that is non-Assur group, is shown in the Figure 1, which is used in many modern wheeled and tracked excavators that are produced by various companies. The module contains a hydraulic cylinder 1 with a rod 2 and a rocker arm 3, to which similar modules can be connected in series. For the most of known types of excavators, the kinematic scheme of the bucket movement includes three such modules.

For example, a single-bucket excavator that is made by Doosan is shown in the Figure 2. There are three rods with hydraulic cylinders. Thus, the drive mechanism of a bucket contains three nonAssur structural groups.

The cavity of the hydraulic cylinder 1 (Fig. 1a) is pressurized with the fluid and the piston moves up from the initial position along the axis of the cylinder together with the rod 2. In the Figure. $1 b$ the arbitrary position of the hydraulic cylinder is shown. In this case the driving link is rod 2 (piston). Since it is impossible to distinguish Assur groups in such mechanism, it is also impossible to use known methods of kinematic analysis of linkage mechanisms [2, 5-7]. Therefore, the problem is to determine the main kinematic characteristics of the mechanism with non-Assur structural groups. The kinematic analysis always precedes the force analysis and it is a necessary step in the study of the dynamic characteristics of mechanical systems.
Thus, first of all, we must determine the position of the group's links. The position of the module when there is no pressure in the hydraulic cylinder is shown in the Figure 1a. We consider the geometry of the module to be known, i.e. the dimensions $a, b, l_{A B}$ and $l_{O A_{0}}$. The stroke $H$ of the rod 2 relative to the hydraulic cylinder 1 is determined by the designer and we also consider it as a known one. The time $T$ of the rod movement on the stroke $H$ is also known. All calculations are carried out in a dimensionless form in relation to the stroke value $H$. For this purpose, we make the following designations:

$$
\begin{gathered}
\lambda_{a}=a / H, \lambda_{b}=b / H, \lambda_{A B}=l_{A B} / H, \\
\lambda_{A O_{0}}=l_{A O_{0}} / H .
\end{gathered}
$$

The distance $l_{O A}$ between the kinematic pair $O$ of hydraulic cylinder and the kinematic pair $A$ of the $\operatorname{rod} 2$ is permanently changing, and in the invariant (dimensionless) form can be calculated as:

$$
\lambda_{O A}=\lambda_{O A_{0}}+a_{k},
$$

where $a_{k}$-a displacement invariant of the law of the rod movement relative to the hydraulic cylinder.

From the triangle $\triangle O B B_{1}$, the angle $\gamma_{2}$ can be defined as: $\gamma_{2}=\operatorname{arctg}\left(\lambda_{a} / \lambda_{b}\right)$.

From the triangle $\triangle O A_{0} B$ we can also define the angle $\gamma_{1}: \gamma_{1}=\arccos \frac{\lambda_{O B}^{2}+\lambda_{A B}^{2}-\lambda_{O A}^{2}}{2 \lambda_{O B} \lambda_{A B}}$.

Angle $\varphi_{3}$ of inclination of the rocker arm 3 to the abscissa axis $x$ :

$$
\varphi_{3}=-90^{\circ}+\gamma_{1}+\gamma_{2}
$$

From the triangle $\triangle O A_{0} B$, the angle $\alpha$ can be also defined: $\alpha=\arccos \frac{\lambda_{O B}^{2}+\lambda_{O A}^{2}-\lambda_{A B}^{2}}{2 \lambda_{O B} \lambda_{O A}}$.

Angle $\varphi_{1}$ of inclination of the cylinder axis to the abscissa axis $x$ can be defined as following:

$$
\varphi_{1}=90^{\circ}+\gamma_{2}-\alpha
$$

Thus, the position of the module links is fully determined. In order to get the initial angles $\varphi_{10}, \varphi_{30}$ of inclination of the links, it is necessary to substitute $a_{k}=0$ in the dependence (2).

In order to determine the kinematic characteristics of the module, it is an important task to define the motion law $a_{k}$ of the rod in relation to the hydraulic cylinder. Obviously, at the beginning of the movement of the rod, its displacement 
a)

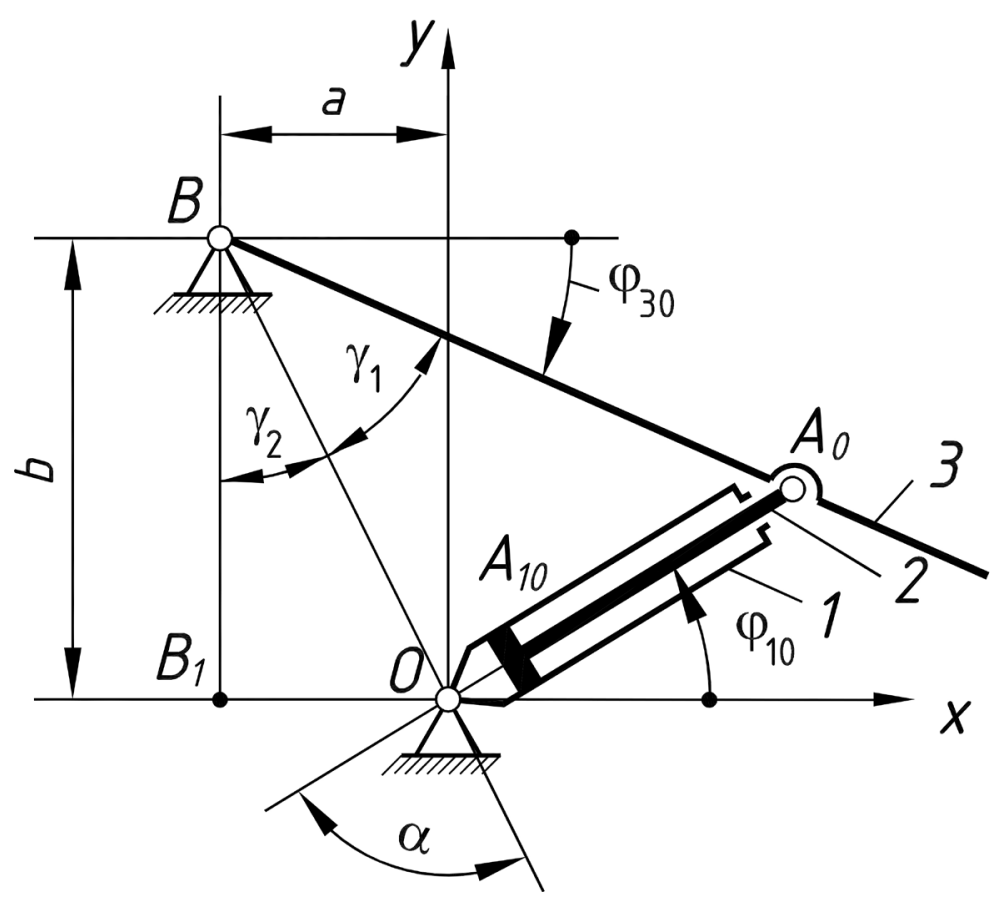

b)

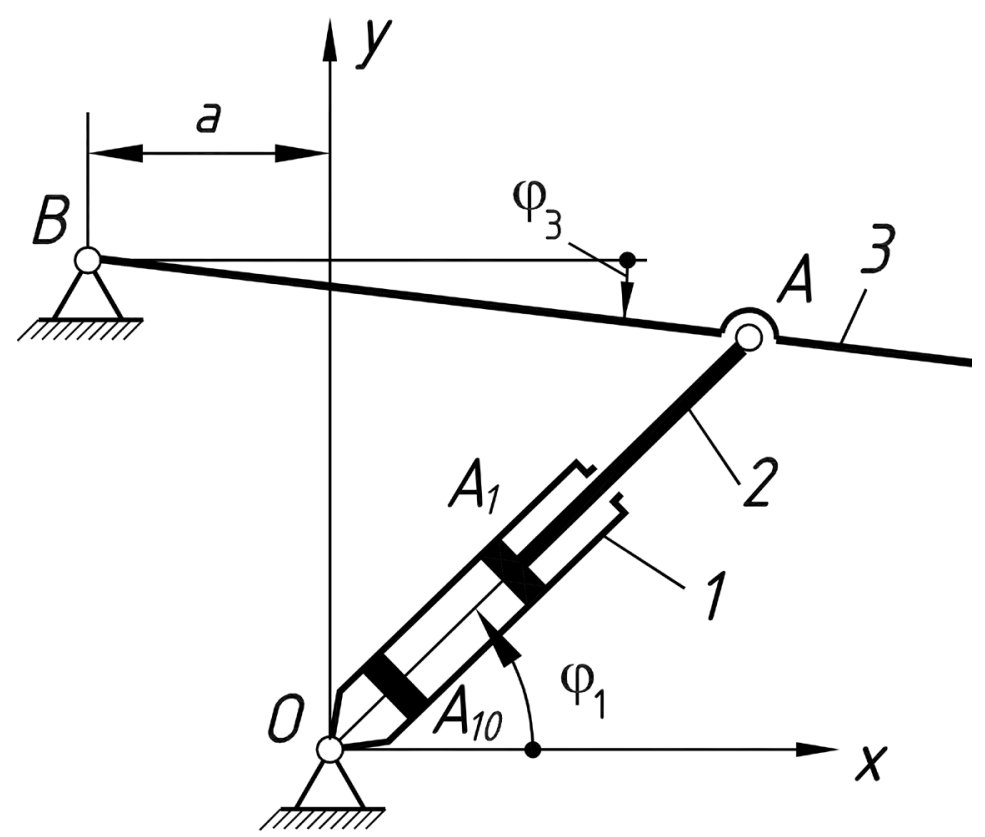

Fig. 1. Non-Assur structural group with the internal driving link 2.

$a$ - initial position, $b$ - arbitrary position; 1 - hydraulic cylinder, 2 - rod, 3 - rocker arm

and speed are zero. At the end of the movement the rod displacement equals to the stroke value $H$, and the speed also equals to zero. Thus, we have four boundary conditions for the rod movement. Therefore, the simplest law of the motion can be represented as a polynomial of the third degree:

$$
a_{k}=a_{0}+a_{1} k+a_{2} k^{2}+a_{3} k^{3},
$$

where $k=0 \ldots 1$ - dimensionless time; coefficients $a_{i}$ can be defined using boundary conditions according to the value of $k$ :

$$
\left.\begin{array}{l}
k=0 \rightarrow a_{k}=0, b_{k}=d a_{k} / d k=0, \\
k=1 \rightarrow a_{k}=1, b_{k}=d a_{k} / d k=0,
\end{array}\right\}
$$

where $b_{k}$-is an invariant of the rod speed. 


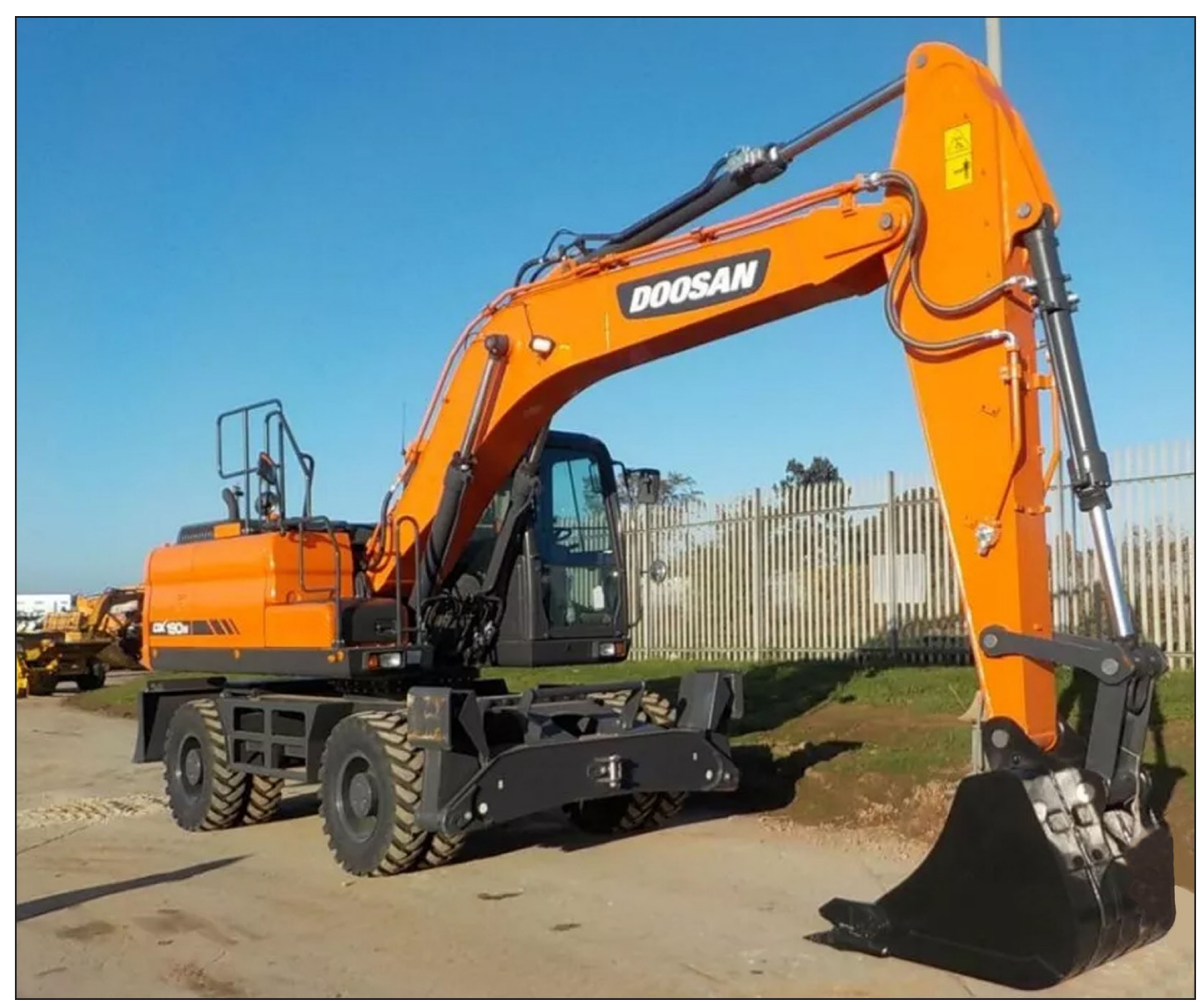

Fig. 2. Doosan single-bucket wheeled excavator

If substitute the boundary conditions (6) to the equation (5), we can obtain a system of two algebraic equations with unknown coefficients $a_{2}$ and $a_{3}$ :

$$
\left.\begin{array}{l}
a_{2}+a_{3}=1, \\
2 a_{2}+3 a_{3}=0
\end{array}\right\}
$$

Taking into account that the coefficients $a_{0}$ and $a_{1}$ are equal to zero, therefore we can obtain a synthesized periodical law of motion (SPL) of the rod relative to the cylinder:

$$
\begin{aligned}
& a_{k}=k^{2}(3-2 k), b_{k}=d a_{k} / d k=6 k(1-k), \\
& c_{k}=d b_{k} / d k=6(1-2 k) .
\end{aligned}
$$

The synthesized periodical law (SPL) is known as the degree law of equally descending acceleration. In the Fig. 3 its kinematic and dynamic $\left(d_{k}\right)$ invariants are shown, where $d_{k}=b_{k} \cdot c_{k}$-invariant value of kinetic power.

Definition of the velocities of the links. It can be considered that the simplest way to get analytical expressions for angular speeds is the usage of Zinoviev method [2] and then differentiate the expressions (3) and (4) by time. However, in that case it is difficult to get concise analytical dependencies.

Since the point $A$ simultaneously belongs to the rocker arm 3 and to the rod 2 (Fig. 1), then we can use a theorem of addition of velocities and accelerations in the relative motion of a point [4], therefore a vector equation that defines the velocity of the point can be written as following:

$$
\vec{v}_{A}=\vec{v}_{A}^{e}+\vec{v}_{A}^{r}
$$

where $v_{A}=\omega_{3} l_{A B}, v_{A}^{e}=\omega_{1} l_{O A}, v_{A}^{r}=b_{k}\left[\frac{H}{T}\right]-$ absolute, bulk and known relative velocities of the point $A, \omega_{1}$ i $\omega_{3}$ - unknown angular velocities of the hydraulic cylinder with the rod and a rocker arm, $T$ - the duration of time when the rod moves to the stroke $H$.

In the Figure 4 the graphical solution of the equation (9) is shown in a certain scale.

A cathetus $p r$ in rectangular triangle $\Delta p r a$ is known: $p r=v_{A}^{r}$. The angle $\varphi^{r}$ can be calculated as following: $\varphi^{r}=\pi / 2+\varphi_{3}-\varphi_{1}$. Then, from that triangle we can determine the unknown velocities $v_{A}, v_{A}^{e}$ and calculate the invariants of angular velocities:

$$
\begin{gathered}
b_{k_{1}}=\frac{b_{k} \operatorname{ctg}\left(\varphi_{1}-\varphi_{3}\right)}{\varphi_{1 \Sigma} \lambda_{O A}}, \\
b_{k_{3}}=\frac{b_{k}}{\varphi_{3 \Sigma} \lambda_{A B} \sin \left(\varphi_{1}-\varphi_{3}\right)} .
\end{gathered}
$$




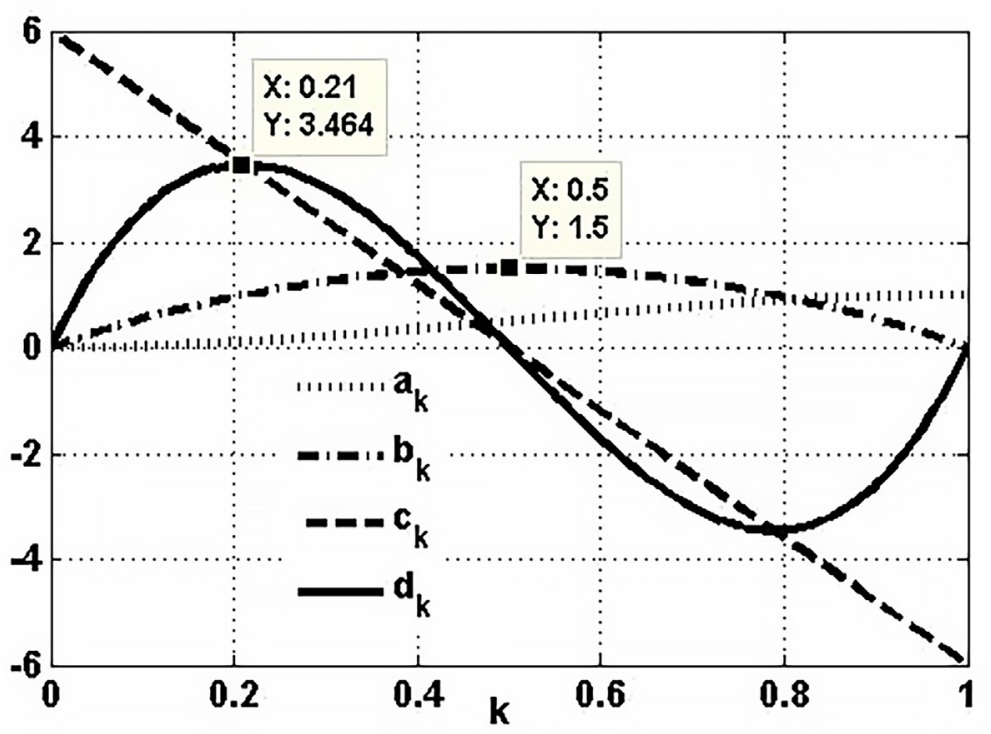

Fig. 3. Invariants of the synthesized motion law of the rod

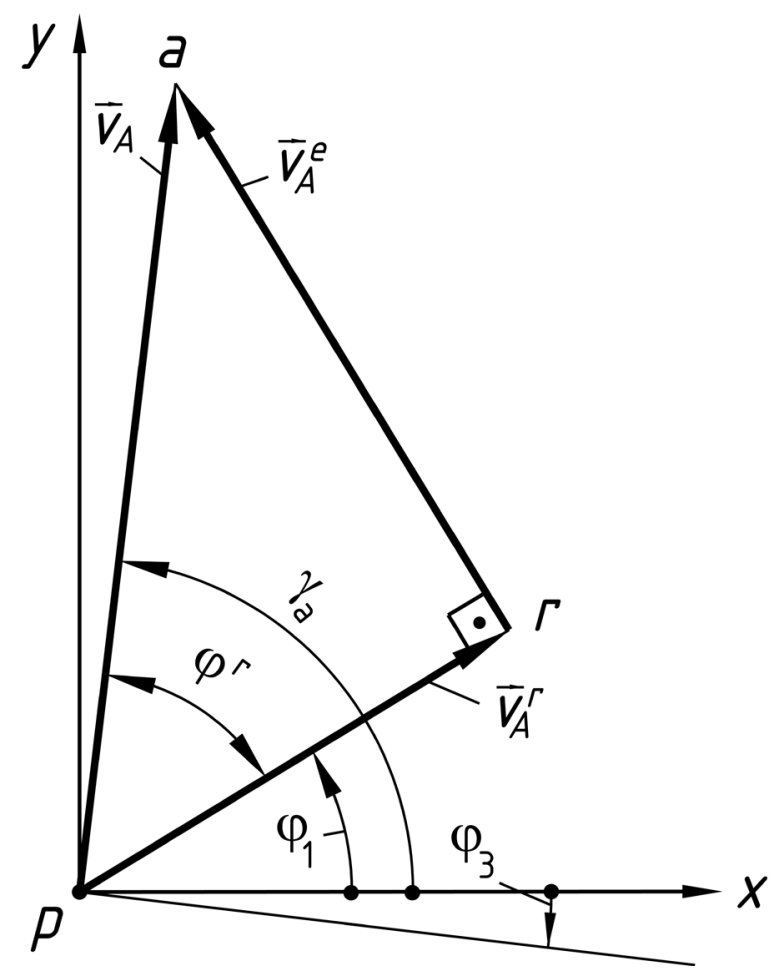

Fig. 4. Geometrically enclosed velocities loop

To get the real values of angular velocities, we must multiply them by the appropriate angular velocity module: $\omega_{1}=b_{k_{1}}\left[\frac{\varphi_{1 \Sigma}}{T}\right], \omega_{3}=b_{\mathrm{k}_{3}}\left[\frac{\varphi_{3 \Sigma}}{T}\right]$,
$\left[\frac{\varphi_{\Sigma}}{T}\right]-$ angular velocity module, $\varphi_{1 \Sigma}$ and $\varphi_{3 \Sigma}$
- the maximum rotation angles of hydraulic cylinder 1 and rocker arm 3.
Definition of the accelerations of the links. To define the angular accelerations of the links, we can make the vector loop closure equation for the acceleration of the point $A$ :

$$
\vec{a}_{A}^{n}+\vec{a}_{A}^{\tau}=\vec{a}_{A}^{e}+\vec{a}_{A}^{r}+\vec{a}_{A}^{k}
$$

where: $a_{A}^{n}=\omega_{3}^{2} l_{A B}$ - the normal acceleration (that is known),

$a_{A}^{\tau}=\varepsilon_{3} l_{A B}-$ the tangential acceleration, $\varepsilon_{3}$ - the unknown angular acceleration of the rocker arm,

$\vec{a}_{A}^{e}=\vec{a}_{A O}^{n}+\vec{a}_{A O}^{\tau}$ - the bulk acceleration, $a_{A O}^{n}=\omega_{1}^{2} l_{O A}$ - the known normal acceleration of point $A$ around the point $O$, $a_{A O}^{\tau}=\varepsilon_{1} l_{O A}$ - the tangential acceleration, $\varepsilon_{1}$ - the unknown angular acceleration of a hydraulic cylinder with a rod,

$a_{A}^{r}=a_{k}\left[\frac{H}{T^{2}}\right]$ and $a_{A}^{k}=2 \omega_{3} b_{k}\left[\frac{H}{T}\right]-$ the known relative and Coriolis accelerations of the point $A$.

Thus, in the equation (11) we have two unknown values of $\varepsilon_{1}$ and $\varepsilon_{3}$, that can be defined by making a projection on the axes of the right coordinate system $x O y$. After certain mathematical transformations, we obtained the following expressions for calculating of the invariants of angular accelerations:

$$
c_{k_{1}}=\frac{K_{1}-K_{2}-K_{3}+K_{4}}{\varphi_{1 \Sigma} \lambda O A} \sin \left(\varphi_{1}-\varphi_{3}\right),
$$




$$
\begin{aligned}
& \text { where: } K_{1}=c_{k} \cos \left(\varphi_{1}-\varphi_{3}\right) \text {; } \\
& K_{2}=2 b_{k_{1}} b_{k} \varphi_{3 \Sigma} \sin \left(\varphi_{1}-\varphi_{3}\right) \text {; } \\
& K_{3}=b_{\mathrm{k}_{1}}^{2} \varphi_{1 \Sigma}^{2} \lambda_{O A} \cos \left(\varphi_{1}-\varphi_{3}\right) \text {; } \\
& K_{4}=b_{k_{3}}^{2} \varphi_{3 \Sigma}^{2} \lambda_{A B} \text {. } \\
& c_{k_{3}}=\frac{c_{k}-b_{k_{1}}^{2} \varphi_{1 \Sigma}^{2} \lambda_{O A}+K_{4} \cos \left(\varphi_{1}-\varphi_{3}\right)}{\varphi_{3 \Sigma} \lambda_{A B} \sin \left(\varphi_{1}-\varphi_{3}\right)}
\end{aligned}
$$

To get the real values of angular accelerations, we should multiply them by the appropriate angular acceleration module:

$$
\varepsilon_{1}=c_{k_{1}}\left[\frac{\varphi_{1 \Sigma}}{T^{2}}\right], \varepsilon_{3}=c_{k_{3}}\left[\frac{\varphi_{3 \Sigma}}{T^{2}}\right] .
$$

To illustrate the obtained analytical dependencies, let's analyze the module movement with the following parameters: $H=0.5 \mathrm{~m}, T=5 \mathrm{sec}$, $\lambda_{a}=0.9, \lambda_{b}=1.8, \lambda_{O A_{0}}=1.6, \lambda_{A B}=2.4$. Dimensions can be chosen arbitrarily, but in order to satisfy the condition that at the movement of the rod relative to the hydraulic cylinder, the movement of the hydraulic cylinder and the rocker arm should be the same sign. In the other words, the angles of inclination of the hydraulic cylinder and rocker arm should increase during the whole period. The results of the analysis are shown in the Figure 5.

It should be also noticed that the correctness of the obtained dependences for the calculation of angular velocities and accelerations was checked numerically. Thus, the expressions for the displacements (3) and (4) were differentiated twice and the received values of angular speeds and accelerations were compared with the values that were obtained analytically. On the whole calculated interval the inaccuracy did not exceed $0.2 \%$.

The analysis of the obtained accelerations graphs of the links of non-Assur structural group shows that the accelerations of the hydraulic cylinder and the rocker arm change almost linearly, and qualitatively similar to the change of the acceleration of the synthesized law of motion (Fig. 3). Since at the beginning and at the end of the movement we have a jump of acceleration, so in these extreme positions the so-called soft impact affects the links, which is caused by sudden inertial loads. Although the values of the angular accelerations of the links are not so big, but the weight of the links in modern excavators can reach thousand or more kilograms. In this case, the moment of inertia forces will be significant and can cause undesirable oscillation processes in the whole group, including the hydraulic system.

Therefore, an additional study was carried out with the goal to find such laws of the rod movement that would not have any acceleration jumps at the beginning and at the end of the movement. This could be technically achieved by regulating of the fluid flow into the hydraulic cylinders. Since the hydraulic cylinder and the rocker arm movement invariants are qualitatively similar to the synthesized rod movement law invariants, let's suppose that such similarity will take place for other given rod movement laws. For the analysis we selected cycloidal SPL, in which the acceleration at the displacement boundaries is zero. The formulae for the displacement invariants of mentioned laws are shown below.

FortheShun'slaw [10]: $a_{k}=10 k^{3}-15 k^{4}+6 k^{5}$.

For the Stoddart's law of degrees 4-5-6-7 [9]: $a_{k}=35 k^{4}-84 k^{5}+70 k^{6}-20 k^{7}$.

For the Stoddart's law of degrees 5-6-7-8-9 [9]: $a_{k}=126 k^{5}-420 k^{6}+540 k^{7}-315 k^{8}+70 k^{9}$.

For the polynomial law of degrees 4-5-6-7-89-10-11 [8]:

$$
\begin{aligned}
& a_{k}=-536,64855 k^{11}+2951,567 k^{10}-6982,173 k^{9}+ \\
& 9283,0259 k^{8}-7579,0146 k^{7}+3866,7329 k^{6}- \\
& -1174,5227 k^{5}+172,03307 k^{4} .
\end{aligned}
$$

The invariants of these laws are shown in the Figure 6.

The analysis shows that the law of degrees 4-56-7-8-9-10-11 has the lowest energy consumption for the drive of the piston and the rod. Therefore, it can be recommended according to the minimal necessary drive power. However, to define the optimal SPL, it is also necessary to find out the SPL of hydraulic cylinder and rocker arm. In the Figure 7 and Figure 8 the graphs of invariants for the hydraulic cylinder and rocker arm are shown. Thus, our hypothesis that the rod invariants are qualitatively similar to the ones of hydraulic cylinder and rocker arm invariants for all given laws is confirmed. There are no acceleration jumps at the motion boundaries. Therefore, according to the requirement of minimal power consumption to overcome the inertia forces during movement of the hydraulic cylinder and rocker arm, the best laws are the following: Shun's law and the polynomial law of degrees 4-5-6-7-8-9-10-11.

For the mentioned laws, the constants of kinetic power peaks $D$ are the lowest ones and for the Shun's law equals to $D_{1}=0.9216, D_{3}=1.71$, 
a)

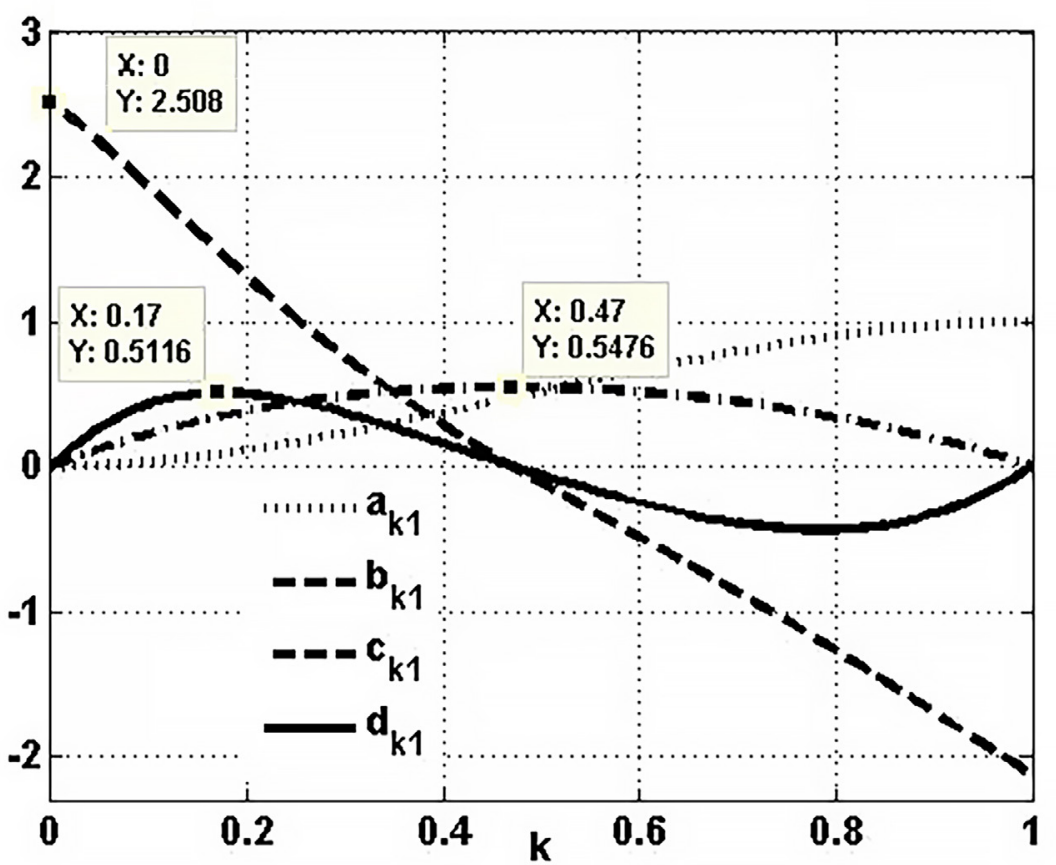

b)

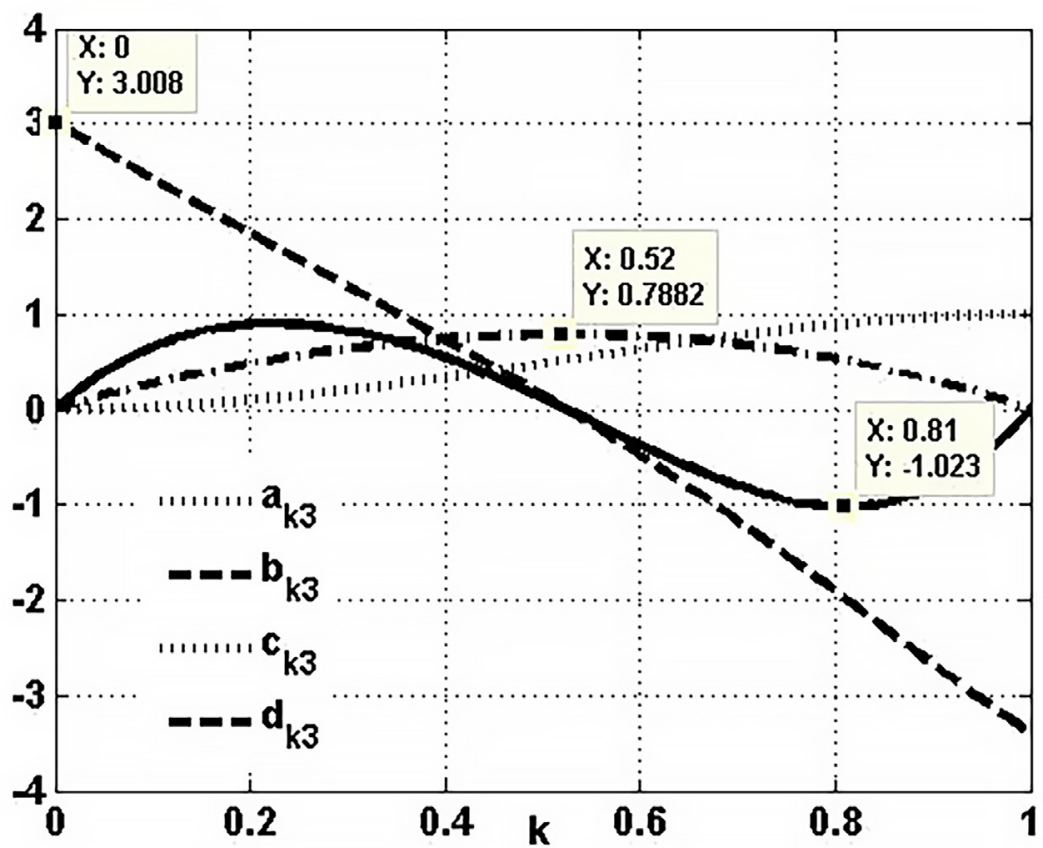

Fig. 5. Invariants in the synthesized SPL:

$a$-invariants for the hydraulic cylinder; $b$ - invariants for the rocker arm.

and for the polynomial law of degrees 4-5-6-78-9-10-11: $D_{1}=0.8591, D_{3}=1.687$. It is established that for the Shun's law the acceleration and the first derivative of the acceleration at the movement boundaries are zero. The same situation is for the polynomial law, but in this case the second derivative is also equal to zero, so the polynomial law of degrees 4-5-6-7-8-9-10-11 is recommended. So, the maximum power to overcome forces of inertia of the rod with a piston, hydraulic cylinder and rocker arm can be calculated using [10]:

$$
\left.\begin{array}{l}
P=D\left[m H^{2} / T^{3}\right] ; \\
P_{1}=D_{1}\left[I_{O} \varphi_{1 \Sigma}^{2} / T^{3}\right] ; \\
P_{3}=D_{3}\left[I_{B} \varphi_{3 \Sigma}^{2} / T^{3}\right],
\end{array}\right\}
$$

where: $m$ - the mass of the rod with the piston, $I_{O}$ i $I_{B}$ - moments of inertia of the 
hydraulic cylinder and the rocker arm with respect to rotation centers $O$ and $B$. This mode is typical for non-working strokes (without loads).

For the unit mass of the rod with the piston and unit moments of inertia, for the chosen data $H=0.5 \mathrm{~m}, T=5 \mathrm{sec}$., the maximum power to overcome the forces of inertia at the movement of the rod, hydraulic cylinder and the rocker arm for different laws, are as shown below:

for the synthesized law $P=3.464\left[0.5^{2} / 5^{3}\right]=6.928 \cdot 10^{-3} \mathrm{~W}$,

$$
P_{3}=1.023\left[0.5276^{2} / 5^{3}\right]=2.278 \cdot 10^{-3} \mathrm{~W} \text {; }
$$

for therecommendedlaw (15)-polynomiallaw ofdegrees 4-11: $P=5.576\left[0.5^{2} / 5^{3}\right]=1.02 \cdot 10^{-2} \mathrm{~W}$; $P_{1}=0.859\left[0.3712^{2} / 5^{3}\right]=9.47 \cdot 10^{-4} \mathrm{~W}$; $P_{3}=1.687\left[0.5276^{2} / 5^{3}\right]=3.76 \cdot 10^{-3} \mathrm{~W}$.

a)

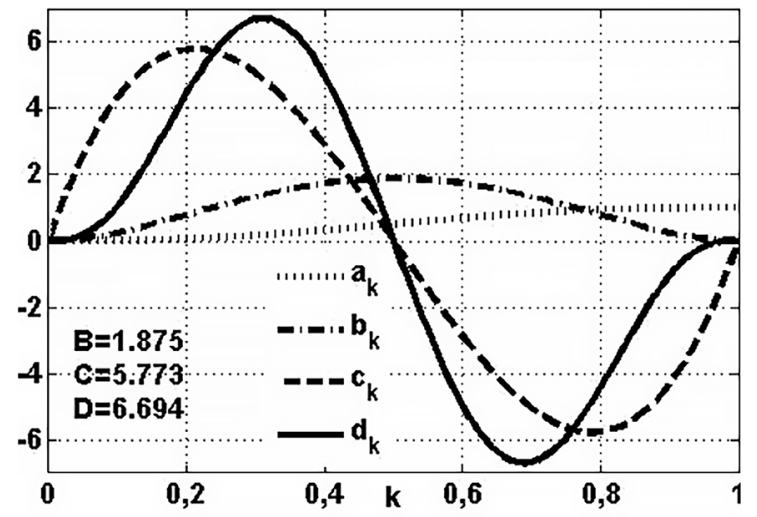

c)

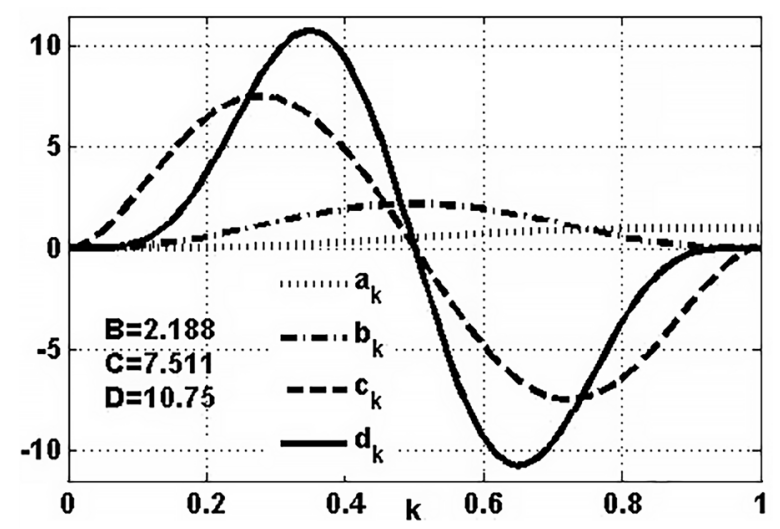

The total load on the rocker arm 3 consists of inertial and static components:

$$
M_{\Sigma}=M_{3_{n}}+M_{s t},
$$

where $\grave{I}_{\text {in }}=I_{O} \varepsilon_{3}=\tilde{n}_{k_{3}}\left[\frac{I_{O} \varphi_{3 \Sigma}}{T^{2}}\right]-$ moment of inertia forces, $M_{s t}$ - the moment of the effective load and gravity. Thus,

$$
\begin{aligned}
& M_{\Sigma}=\left(c_{k_{3}}+\frac{M_{s t}}{\left[I_{O} \varphi_{3 \Sigma} T^{-2}\right]}\right)\left[\frac{I_{O} \varphi_{3 \Sigma}}{T^{2}}\right]= \\
& =\left(c_{k_{3}}+p_{k_{3}}\right)\left[I_{O} \varphi_{3 \Sigma} / T^{2}\right],
\end{aligned}
$$

where $p=\frac{M_{s t}}{\left[I_{O} \varphi_{3 \Sigma} T^{-2}\right]}-$ Newton's number [10]. So, the total power that is used for lifting the load:

$$
P_{3 \Sigma}=\left(c_{k_{3}}+p_{k_{3}}\right) b_{k_{3}}\left[\frac{I_{B} \varphi_{3 \Sigma}^{2}}{T^{3}}\right]=u_{k_{3}}\left[\frac{I_{B} \varphi_{3 \Sigma}^{2}}{T^{3}}\right]
$$

b)

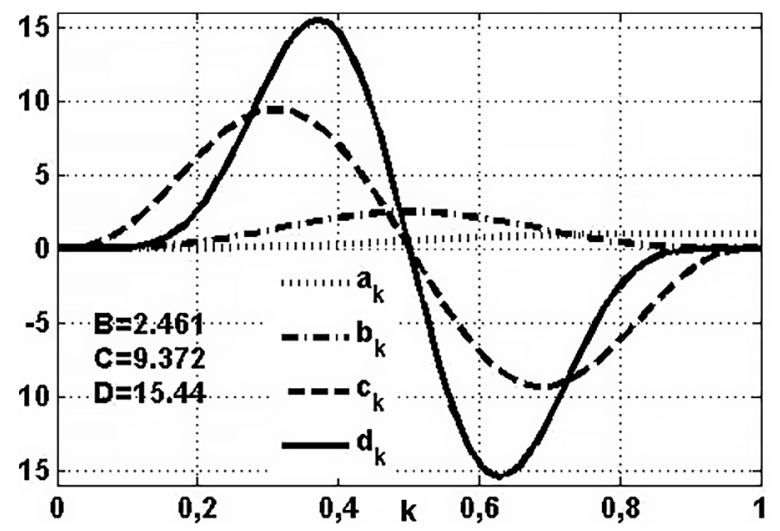

d)

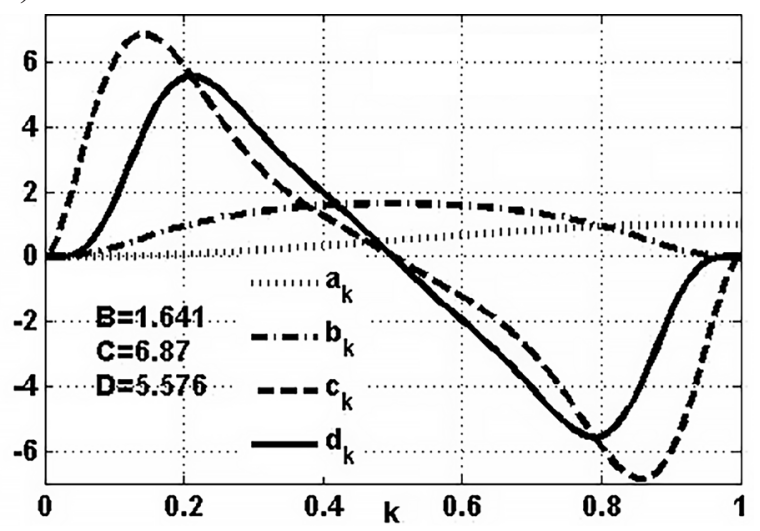

Fig. 6. Invariants for known periodical laws:

$a$ - Stoddart's law, degrees 4-5-6-7; $b$ - Shun's law; $c$ - Stoddart's law, degrees 5-6-7-8-9;

$d$ - Polynomial law of degrees 4-5-6-7-8-9-10-11 
where $u_{k_{3}}=\left(c_{k_{3}}+p_{k_{3}}\right) b_{k_{3}}-$ the total power invariant. Newton's number can be assumed as $p_{k_{3}}=400$ or more for common truck cranes, so inertial moments play a minor role and can be neglected.

Further researches are planned to be carried out in the field of optimization of design parameters and to analyze the mechanisms of known excavators and hydraulic truck cranes.

\section{CONCLUSIONS}

The first stage of dynamic analysis of linkage mechanisms with internal driving link was carried out and analytical dependences for calculation of kinematic characteristics of the links were obtained. It is shown that in case of unregulated movement of the hydraulic drive, there are sudden inertial loads (soft impacts) are taken place on the moving links of the module at the beginning and at the end of the movement, which is undesirable. The research was carried out for the various cycloidal laws of the rod and the piston movement, the obtained results showed that under such conditions the laws of movement of links do not have soft impacts in the extreme positions of the displacement. It is also shown that the power of the hydraulic drive is affected mostly by the static load.

The results of the work can be used to investigate the movement of excavator buckets with the hydraulic drive, boom movement of the hydraulic truck cranes, road construction machines, actuator mechanisms of industrial robots. a)

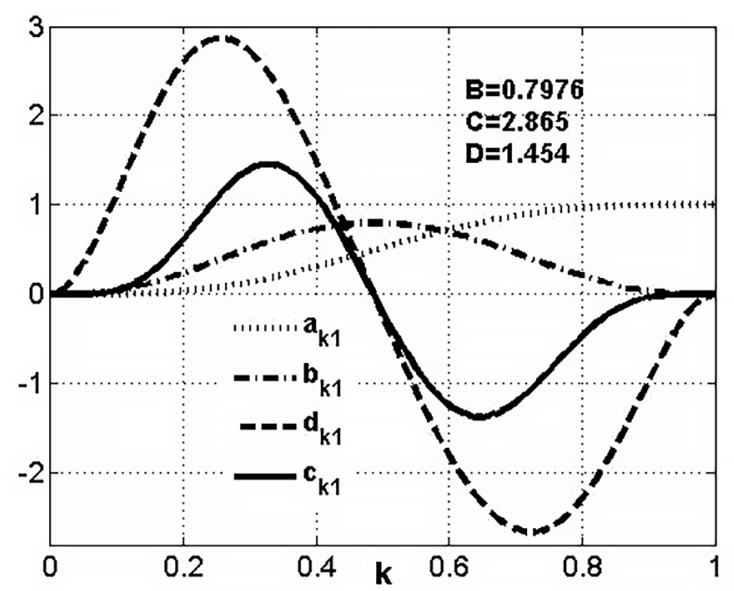

c)

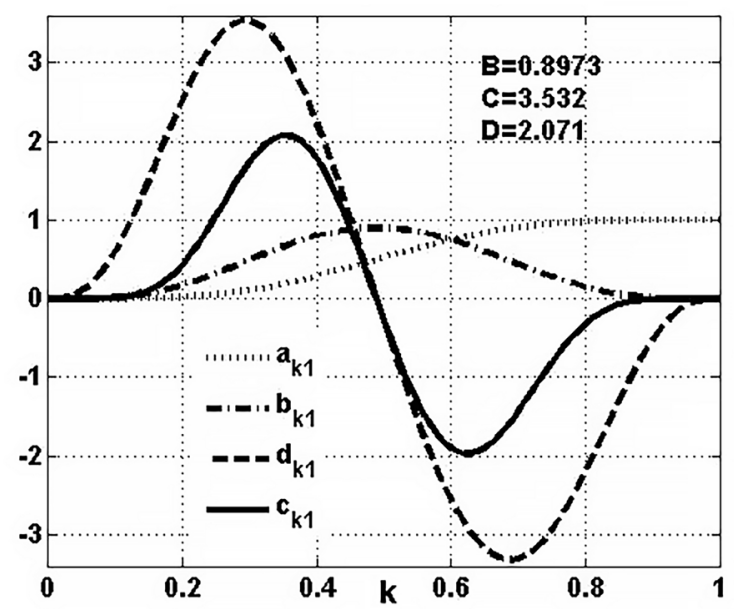

b)

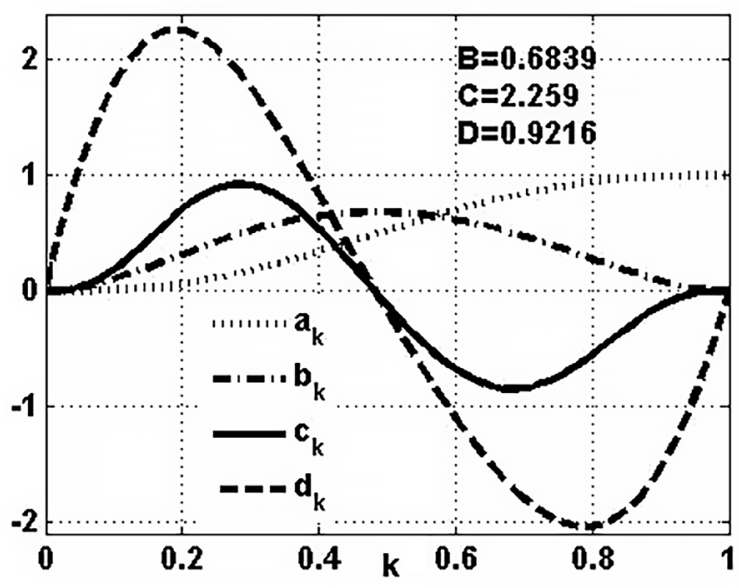

d)

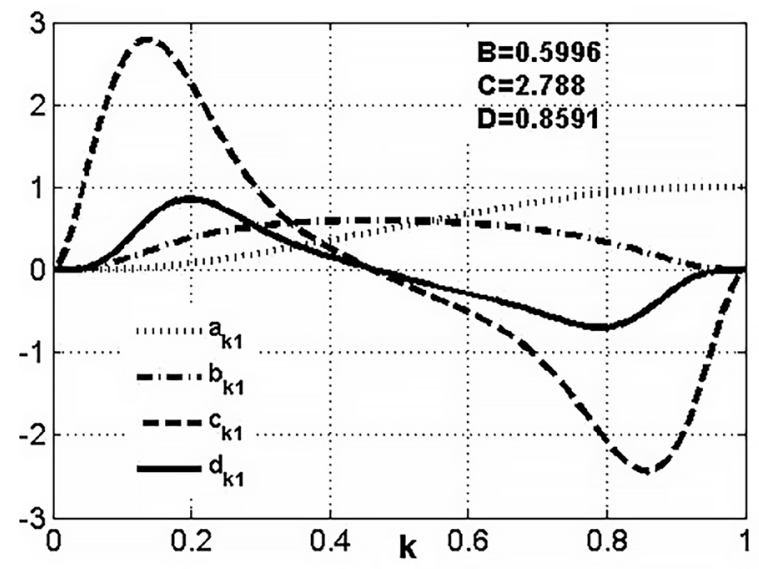

Fig. 7. Invariants of hydraulic cylinder for laws:

$a$ - Stoddart's law, degrees 4-5-6-7; b - Shun's law; $c$ - Stoddart's law, degrees 5-6-7-8-9;

$d$ - Polynomial law of degrees 4-5-6-7-8-9-10-11. 
a)

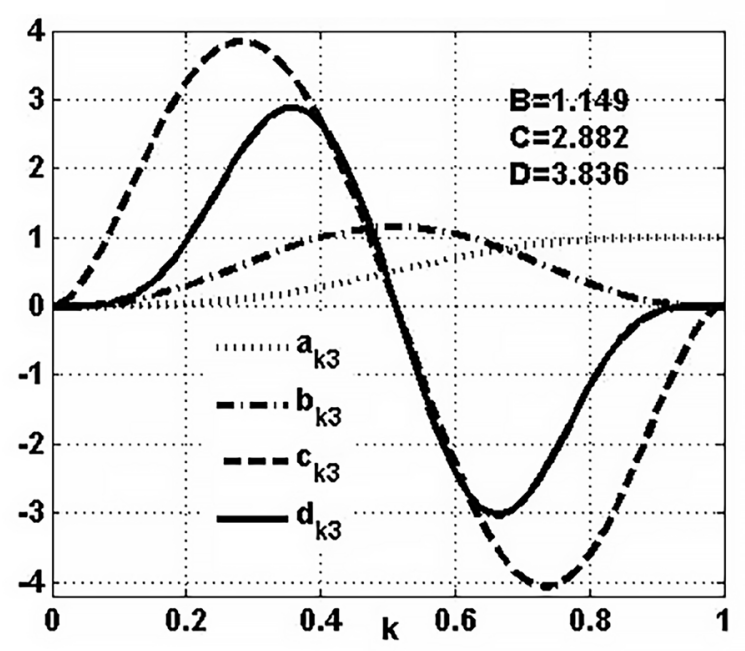

c)

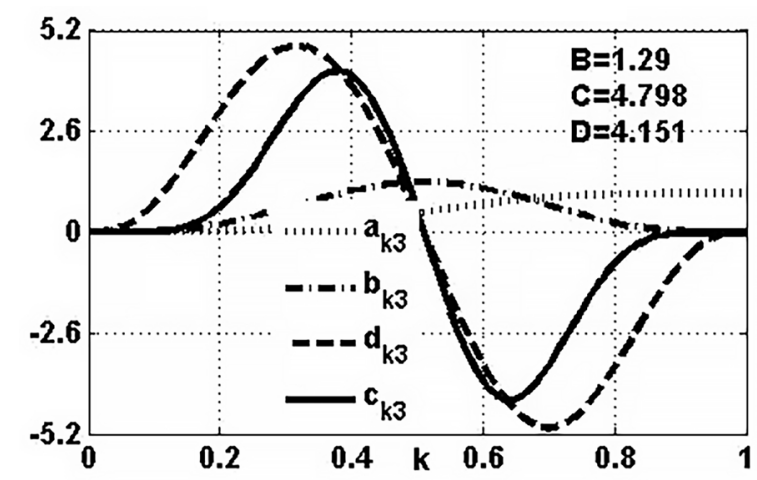

b)

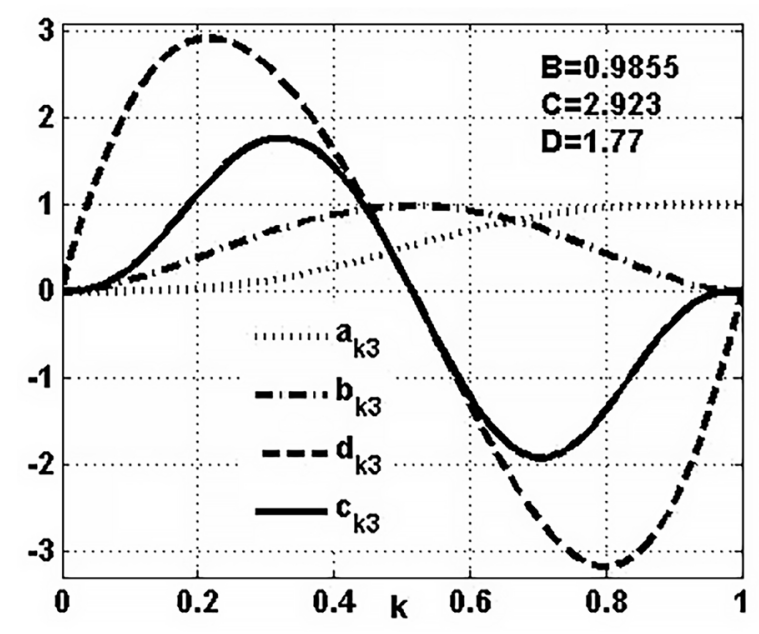

d)

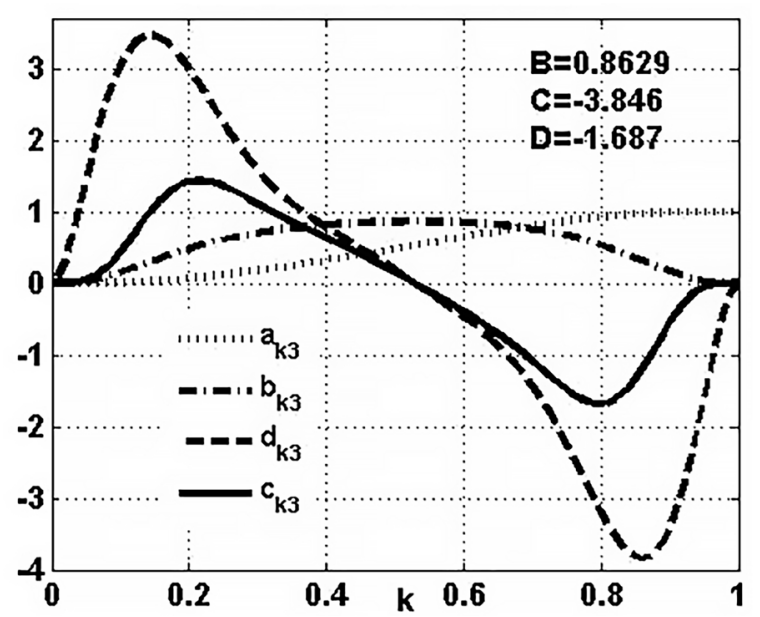

Fig. 8. Invariants of rocker arm for laws:

$a$ - Stoddart's law, degrees 4-5-6-7; $b$ - Shun's law; $c$ - Stoddart's law, degrees 5-6-7-8-9;

$d$ - Polynomial law of degrees 4-5-6-7-8-9-10-11.

\section{REFERENCES}

1. Degrave V.S. Special positions of the planar nonAssur structural groups with internal inputs. Theory of Mechanisms and Machines, 2(8), Vol. 4, 2006, 81-85.

2. Kinytskyi Ya.T. Theory of Mechanisms and Machines: Naukowa Dumka, 2002.

3. Kharzhevskyi V.O. Kinematic synthesis of linkage mechanisms using Burmester points at the given dwell duration of the output link. Advances in Science and Technology Research Journal, 2(11), 2017, 139-145.

4. Kuzio I.V., Vankovych T.N. and Zinko Ya.Z. Theoretical Mechanics. Statics. Kinematics. Rastr-7, 2010.

5. Mata A.S., Torras A.B. and Carrillo J.C. Fundamentals of Machine Theory and Mechanisms. Springer
International Publishing, 2016.

6. McCarthy J. and Soh G. Geometric Design of Linkages, 2nd edition : Springer-Verlag New York, 2011.

7. Norton R.L. Design of Machinery: An Introduction to the Synthesis and Analysis of Mechanisms and Machines: McGraw Hill, 2011.

8. Pasika V.R. Synthesis of optimal laws of periodical motion. Scientific papers of Ukrainian Academy of Printing, 1(9), 2006, 38-44.

9. Stoddart D.A. Polydyne Cam Design, Machine Design, 1953, pp. 121-164.

10. Tir K.V. Mechanics of polygraphic automatic machines, Kniga, 1965.

11. Terenteva A.D. Analysis of the movement accuracy of the single-bucket excavator's working body. Theory of Mechanisms and Machines, 4(32), Vol. 14, 2016, 218-228. 